\title{
Is anomalous scattering typical for pulsars?
}

\author{
Wojciech Lewandowski ${ }^{1}$, Jarosław Kijak ${ }^{1}$, Leszek Błaszkiewicz ${ }^{2,3}$, \\ Karolina Rożko ${ }^{1}$ and Andrzej Krankowski ${ }^{2}$ \\ ${ }^{1}$ Janusz Gil Institute of Astronomy, University of Zielona Góra, \\ ul. Szafrana 2, 65-516 Zielona Góra, Poland \\ email: w.lewandowski@ia.uz.zgora.pl \\ ${ }^{2}$ Space Radio-Diagnostics Research Centre, University of Warmia and Mazury, \\ Prawocheńskiego 9, 10-720 Olsztyn, Poland \\ ${ }^{3}$ Faculty of Mathematics and Computer Sciences, University of Warmia and Mazury, \\ Słoneczna 54, 10-710 Olsztyn, Poland
}

\begin{abstract}
Recent studies of interstellar scattering frequency evolution indicate, that for a large number of pulsars the measured scatter time to frequency scaling does not follow predictions based upon simple scattering models. The number of such deviations suggests, that what was previously known as "anomalous scattering" is actually quite normal for a large subset of the pulsar population. New observations are needed, especially at low frequencies, to study the scattering of low-DM objects. In this regard even small telescopes, such as single LOFAR stations - like PL-612 in Bałdy, near Olsztyn, Poland - can be extremely helpful.
\end{abstract}

Keywords. pulsars: general, ISM: genera

The temporal and angular broadening of the pulsar profiles is caused by the scattering of the radio waves emitted by a pulsar. This, along with other effects such as an interstellar dispersion of the signal, as well as the interstellar scintillations, happens in the ionized interstellar medium (ISM). The current models of the interstellar scattering predict that the frequency dependence of the observed parameters is closely bound to the density fluctuations caused by turbulent ISM. Most of the models assume, that the turbulence spectrum in the ISM is the same as the Kolmogorov's spectrum, however there is increasing observational evidence that suggests otherwise (see Lewandowski et al. 2013, Lewandowski et al. 2015a, Lewandowski et al. 2015b).

The estimates of the scatter time frequency scaling index a may be used to ascertain the spectrum of the turbulence in the ionised interstellar medium. Simplest models predict, that for the thin-screen scattering the frequency scaling index $\alpha$ should be between 4.0 and 4.4 (the later is the value for Kolmogorovs turbulence).

Löhmer et al. (2001) and Löhmer et al. (2004) noticed that for large DM pulsars the values of a tend to be much lower, which can possibly be explained only by the existence of multiple scattering screens or anisotropy effects. In our work (Lewandowski et al. 2013, Lewandowski et al. 2015a, Lewandowski et al. 2015b) we have shown that such deviations from the model are common for pulsars with all DM values, including the closest ones. Hence the term "anomalous scattering" does no longer apply, as the number of pulsars that seem to behave this way is steadily growing (see also Krishnakumar et al., 2017 and Geyer et al. 2017).

The phenomenon of scattering is still not well studied for the closest, low-DM pulsars, as the estimates of pulse broadening for these objects require low frequency observations. For a sample of strong pulsars even the small telescopes such as a single LOFAR station may be succesfully used for interstellar scattering observations. Therefore we started an observing project using the PL-612 station. It is one of the three polish LOFAR stations, 

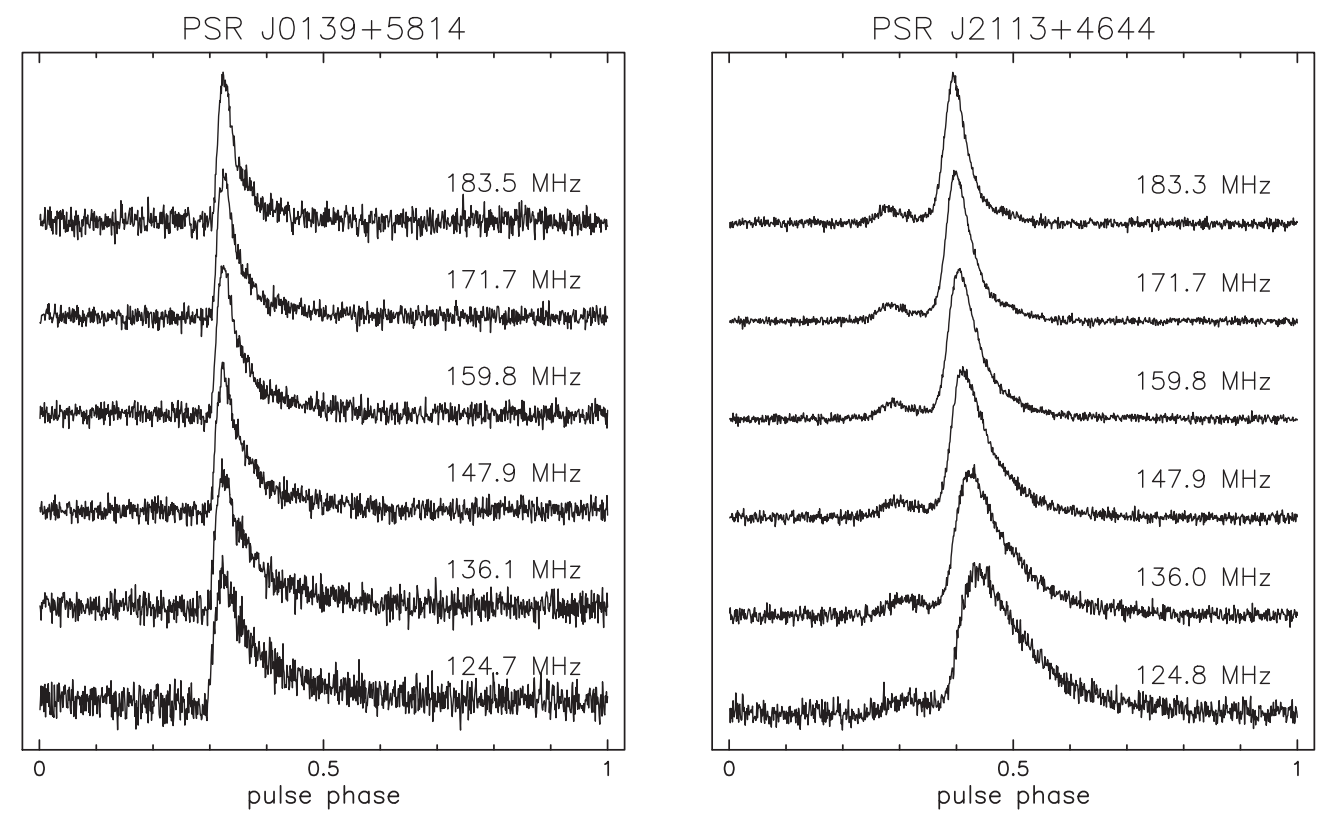

Figure 1. Pulse broadening frequency evolution observed within the LOFAR HBA-low band for two pulsars. Data from 2-hour integration with the PL-612 station in Bady, near Olsztyn.

and its located in Bałdy near Olsztyn (see Błaszkiewicz et al. 2015). The station is maintained by the University of Warmia and Mazury. The data acquisition system is based on the one employed by the German LOFAR Consortium (GLOW).

First pulsar data were gathered in the summer of 2016, and the regular pulsar observations started in autumn. The observations are conducted at the frequency of about 150 $\mathrm{MHz}$, with $72 \mathrm{MHz}$ bandwidth. This gives us a large fractional bandwidth, which means that the frequency evolution of scattering is observable within the band (see the examples in Figure 1). Splitting the bandwidth into several sub-bands allows us to estimate the frequency scaling slope.

\section{Acknowledgements}

We thank to the Polish Ministry of Science and Higher Education for granting funds for construction of three polish LOFAR stations (6339/IA/158/2013.1), Polish contribution to the ILT (DIR/WK//2016/05) and for maintenance of the LOFAR PL-612 Bałdy (220815/E-383/SPUB/2016/2). PSNC in Poznań, OLMAN in Olsztyn and Computer Center in Zielona Góra are responsible for the IT and Network service. The work was also partially supported by Polish National Science Centre (DEC-2013/09/B/ST9/02177).

\section{References}

Błaszkiewicz, L., Lewandowski, W., Krankowski, A., Kijak, J., Koralewska, O., \& Dabrowski, B. 2015, Acta Geophisica, 64, 1

Geyer, M., Karastergiou, A., \& Kondratiev, V. I. et al. 2017, MNRAS, 470, 2659

Krishnakumar, M. A., Joshi, B. C., \& Manoharan, P. R. 2017, ApJ, 846, 104

Löhmer O., Kramer M., Mitra D., Lorimer D. R. \& Lyne A. G. 2001, ApJ, 562, L157

Löohmer O., Mitra D., Gupta Y., Kramer M., Ahuja A. 2004, A\& A, 425, 569

Lewandowski, W., Dembska, M., Kijak, J., \& Kowalińska, M. 2013, MNRAS, 434,69

Lewandowski, W., Kowalińska, M., \& Kijak, J. 2015a, MNRAS, 449,1570

Lewandowski, W., Rożko, K., Kijak, J., \& Bhattacharyya, B.; Roy, J. 2015b, MNRAS, 454,2517 